\title{
Smooth muscle cell matrix
}

\section{metalloproteinases in idiopathic pulmonary arterial hypertension}

\author{
H. Lepetit*, S. Eddahibi*, E. Fadel ${ }^{\#}$, E. Frisdal ${ }^{\top}$, C. Munaut ${ }^{+}$, A. Noel ${ }^{+}$, M. Humbert ${ }^{\S}$, \\ S. Adnot*, M-P. D’Ortho* and C. Lafuma*
}

ABSTRACT: Pulmonary arterial hypertension (PAH) results from persistent vasoconstriction, smooth muscle growth and extracellular matrix (ECM) remodelling of pulmonary arteries (PAs). Matrix metalloproteinases (MMPs) are matrix-degrading enzymes involved in ECM turnover, and in smooth muscle cell (SMC) and endothelial cell migration and proliferation. MMP expression and activity are increased in experimental PAH. Therefore, this study investigated whether similar changes occur in idiopathic PAH (IPAH; formerly known as primary pulmonary hypertension).

Both in situ and in vitro studies were performed on PAs from patients undergoing lung transplantation for IPAH and from patients treated by lobectomy for localised lung cancer, who served as controls.

In IPAH, MMP-tissue inhibitor of metalloproteinase (TIMP) imbalance was found in cultured PASMCs, with increased TIMP-1 and decreased MMP-3. MMP-2 activity was markedly elevated as a result of increases in both total MMP-2 and proportion of active MMP-2. In situ zymography and immunolocalisation showed that MMP-2 was associated with SMCs and elastic fibres, and also confirmed the MMP-3-TIMP-1 imbalance.

In conclusion, the findings of this study were consistent with a role for the matrix metalloproteinase-tissue inhibitor of metalloproteinase system in pulmonary vascular remodelling in idiopathic pulmonary arterial hypertension. The matrix metalloproteinase-tissue inhibitor of metalloproteinase imbalance may lead to matrix accumulation, and increased matrix metalloproteinase-2 activity may contribute to smooth muscle cell migration and proliferation. Whether these abnormalities are potential therapeutic targets deserves further investigation.

KEYWORDS: Extracellular matrix, idiopathic pulmonary arterial hypertension, matrix metalloproteinases, smooth muscle cells, tissue inhibitor of matrix metalloproteinase

$\mathbf{P}$ ulmonary arterial hypertension $(\mathrm{PAH})$ is characterised by a progressive increase in pulmonary vascular resistance, which ultimately leads to right ventricular failure. Idiopathic PAH (IPAH) is a clinical term used to describe a rare and fatal condition for which no underlying cause can be found. The typical pulmonary artery (PA) lesions in IPAH include adventitial thickening, medial hypertrophy, neointima formation and plexiform lesions in the lung vascular bed. Cell proliferation and extracellular matrix (ECM) accumulation are prominent [1]. An important component of these changes is ECM remodelling, which results from a complex interplay between the synthesis and proteolysis of ECM constituents.

For editorial comments see page $\mathbf{7 7 8}$.
Early studies involving ultrastructural evaluation of PAs in lung biopsies from patients with PAH showed fragmentation of the internal elastic lamina [2], suggesting a role for an elastinolytic enzyme in the pathophysiology of the disease. In agreement with this hypothesis, animal studies showed both an early increase in elastinolytic activity preceding the development of vascular changes and a later increase associated with disease progression. Moreover, serine-elastase inhibitors have been found to induce regression of experimental toxic PAH [3,4]. Although these findings emphasise the major contribution of serine-elastase to the pathogenesis of $\mathrm{PAH}$, matrix metalloproteinases (MMPs) may also play a role.

MMPs are a family of matrix-degrading proteases that are categorised according to structural

\section{AFFILIATIONS}

*INSERM U 492, Dépt de

Physiologie, CHU Henri Mondor Créteil, Créteil,

\#UPRES EA2705, Service de

Chirurgie Thoracique, Vasculaire et de Transplantation Cardiopulmonaire, Hôpital Marie Lannelongue, Le Plessis-Robinson,

•INSERM U 551, Hôpital de la Pitié, Paris, and

sUPRES EA2705, Service de

Pneumologie Hôpital A. Béclère, APHP, Clamart, France.

+Laboratoire de Biologie des

Tumeurs et du Développement, Université de Liège, Sart Tilman,

Liège, Belgium.

CORRESPONDENCE

H. Lepetit

INSERM U492

Dépt de Physiologie

Faculté de Médecine

CHU Henri Mondor Créteil

94010 Créteil

France

Fax: 33148981777

E-mail:

helene.lepetit@creteil.inserm.fr

Received:

June 162004

Accepted after revision:

January 102005

\section{SUPPORT STATEMENT}

This study was supported by the Institut National de la Santé Et de la Recherche Médicale (INSERM). H. Lepetit received a studentship from the French Ministry of Education, Research, and Technology (Paris, France). 
similarities [5]. MMPs are also classified based on substrate specificity into collagenases (which degrade fibrillar collagen), stromelysins, membrane type (MT)-MMPs (which have a broad spectrum of activity) and gelatinases (which cleave basement membrane components). Most MMPs are secreted into the extracellular milieu as inactive proteins. MMP activity is modulated by tissue inhibitors of metalloproteinases (TIMPs), of which four forms have been identified [5].

Studies in experimental models have sought to determine whether MMPs are involved in $\mathrm{PAH}$ progression and contribute to smooth muscle cell (SMC) migration. Several studies concluded that MMPs contributed to PAH progression in animals [3, 6-8], but the effects of MMP inhibitors differed across models. In addition, little is known about the MMPTIMP balance in humans with IPAH. To address this question, the current authors conducted studies on both cultured PASMCs and tissue samples from patients with IPAH and from controls.

Since IPAH is characterised by accumulation of ECM components, most notably collagen, the present authors studied the MMP inhibitors TIMP-1 and TIMP-2, as well as the collagenase MMP-1. MMP-3 and MMP-7 were also evaluated, given the ability of these enzymes to degrade a broad range of substrates [5]. In addition, the authors studied gelatinases (MMP-9 and MMP-2) and the MMP-2 activators MT1-MMP and MT2-MMP [9], which are involved in SMC migration and elastin degradation [10].

\section{MATERIALS AND METHODS Sample collection}

Studies were performed on PAs $(0.5-1 \mathrm{~cm}$ in diameter), obtained during lung transplantation in five patients with IPAH and during lobectomy for localised lung cancer in six controls. The mean pulmonary artery pressure in the group with IPAH was $72 \pm 12 \mathrm{mmHg}$. None of the patients had detectable type-II bone morphogenetic protein (BMP) receptor (BMPR-II) gene mutations, which are known to play a key role in familial PAH. PAs were either prepared for in situ studies then frozen in liquid nitrogen or prepared for cell culture studies.

\section{Culture and treatment of human PA-SMC}

PA-SMCs, as cultured from transplants as previously described [11, 12], were used between passages 2 and 4 . Trypsinised PA-SMCs were seeded at a density of $4 \times 10^{4}$ cells $\cdot \mathrm{cm}^{-2}$ in Dubelcco's modified eagle's medium, containing
$10 \%$ foetal bovine serum, then grown for $24 \mathrm{~h}$. The cells were then left in serum-free medium (SFM) for $24 \mathrm{~h}$. For the next $24 \mathrm{~h}$, the cells were exposed to fresh SFM containing one of the following: $5 \mathrm{ng} \cdot \mathrm{mL}^{-1}$ or $10 \mathrm{ng} \cdot \mathrm{mL}^{-1}$ transforming growth factor (TGF)- $\beta ; 10 \mathrm{ng} \cdot \mathrm{mL}^{-1}$ platelet-derived growth factor (PDGF); $50 \mathrm{ng} \cdot \mathrm{mL}^{-1} \mathrm{BMP}-4 ; 200 \mathrm{ng} \cdot \mathrm{mL}^{-1} \mathrm{BMP}-6$ (R\&D Systems, Europe Ltd, Abingdon, UK); or $1 \times 10^{-7} \mathrm{M}$ phorbol-12-myristate-13acetate (PMA; Sigma, St Quentin-Fallavier, France). SFM alone was used for the control cells. Conditioned media or RNA samples were collected after $24 \mathrm{~h}$ and stored at $-80^{\circ} \mathrm{C}$.

\section{ELISAs}

ELISA kits (Biotrak ${ }^{\mathrm{TM}}$; Amersham Pharmacia Biotech, Orsay, France) were used according to the manufacturer's instructions. The kits detect: total MMP-1; total MMP-3; total TIMP-1; total TIMP-2 except TIMP-2 complexed with latent MMP-2; latent MMP-2 only (whether complexed with TIMP-2 or not); and latent MMP-7. The data were expressed as the amount of protein produced in $24 \mathrm{~h}$ by $1 \times 10^{5}$ cells. Assays were performed in triplicate for four IPAH patients and four controls.

\section{Reverse and gelatin zymography}

Reverse and gelatin zymographies were done as previously described [8]. For reverse zymography, 11\% SDS-polyacrylamide gel, impregnated with both $0.05 \%$ gelatin and gelatinaserich solution, was used. After Coomassie brilliant-blue staining, inhibitors of gelatin-degrading enzymes were identified as blue zones against a clear background. Molecular masses were estimated using both pre-stained standard molecular markers and recombinant human (rh) TIMP-2 (Valbiotech/Abcys, Paris, France). The amounts of TIMP-2 in samples were estimated by comparison with a standard curve obtained using rhTIMP-2 and results given per $1 \times 10^{5}$ cells. For zymography, MMP-2 (both latent and active) was assessed semi-quantitatively for five patients with IPAH and five controls using gel scanning with Bio-imaging system and densitometry with GeneTools (SynGene, Ozyme, France).

\section{RT-PCR}

Total RNA was extracted from cultured cells from seven patients with IPAH and eight controls, using Trizol ${ }^{\circledR}$ reagent (Life Technologies, Cergy-Pontoise, France), as recommended by the manufacturer. The concentration of extracted RNA was determined spectrophotometrically at $260 \mathrm{~nm}$. The RT-PCR reactions for quantitative studies were performed as previously described by LAMBERT et al. [13] using specific pairs of

TABLE 1 Sequence of forward and reverse primers used for quantitative RT-PCR amplification

Forward primers $\left(5^{\prime}-3^{\prime}\right)$

Reverse primers $\left(5^{\prime}-3^{\prime}\right)$

\section{MMP-1}

MMP-2

MT1-MMP

TIMP-1

TIMP-2
GAGCAAACACATCTGAGGTACAGGA AGATCTTCTTCTTCAAGGACCGGTT GGATACCCAATGCCCATTGGCCA CATCCTGTTGTTGCTGTGGCTGAT CTCGCTGGACGTTGGAGGAAAGAA
TTGTCCCGATGATCTCCCCTGACA GGCTGGTCAGTGGCTTGGGGTA CCATTGGGCATCCAGAAGAGAGC GTCATCTTGATCTCATAACGCTGG AGCCCATCTGGTACCTGTGGTTCA

MMP: matrix metalloproteinase; MT: membrane type; TIMP: tissue inhibitor of metalloproteinase 


\begin{tabular}{lccc} 
TABLE 2 & $\begin{array}{l}\text { Number of cycles, mssRNA type and number of } \\
\text { copies for quantitative RT-PCR }\end{array}$ \\
& Cycles $\mathbf{n}$ & mssRNA & mssRNA copies $\mathbf{n}$ \\
\hline MMP-1 & 30 & $\mathrm{C} 3$ & $5 \times 10^{4}$ \\
MMP-2 & 28 & $\mathrm{C} 1$ & $1 \times 10^{5}$ \\
MT1-MMP & 30 & $\mathrm{C} 1$ & $5 \times 10^{4}$ \\
TIMP-1 & 26 & $\mathrm{C} 4$ & $5 \times 10^{6}$ \\
TIMP-2 & 26 & $\mathrm{C} 4$ & $2 \times 10^{6}$ \\
$\mathbf{2 8 s}$ & 18 & $\mathrm{C} 1$ & $5 \times 10^{5}$ \\
\hline
\end{tabular}

mssRNA: multiple synthetic internal stranded RNA; MMP: matrix metalloproteinase; MT: membrane type; TIMP: tissue inhibitor of metalloproteinase.

primers (table 1). Table 2 shows the number of cycles and the type and number of multiple synthetic internal standard (mss) RNAs. Nonquantitative RT-PCRs for MMP-3, MMP-7, MMP-9, MMP-13 and MT2-MMP were performed as described above but without mssRNA. The primers and PCR cycle conditions are listed in tables 3 and 4 .

\section{Immunofluorescence on culture cells}

Antibodies against MMP-3, MT1-MMP, TIMP-1 and TIMP-2 were goat polyclonal antibodies (Santa Cruz Biotechnology, Tebu, France), and the antibody against MMP-2 was a rabbit polyclonal antibody (Chemicon, Temacula, CA, USA). Primary antibodies (1:200) were revealed with fluorescein isothiocyanate-conjugated anti-goat (Santa Cruz Biotechnology) or anti-rabbit (Biomeda Microm, Frachville, France) antibodies diluted 1:1,000. The cells were then treated for nuclear labelling with bisbenzimide dye (Hoechst-33258; Sigma, Paris, France). Finally, the cells were observed under an immunofluorescent inverted microscope (Axiovert-200M; Carl-Zeiss-Vision, Rueil-Malmaison, France). Quantification was realised using ImageJ 1.32J (Wayne Rasband, Maryland, $\mathrm{NH}, \mathrm{USA})$ on photographs $(\mathrm{n}=4)$ taken at a magnification of $\times 600$.

\section{Immunostaining and gelatinolytic activity in PA}

MMP-2, MMP-3 and TIMP-1 in PAs were localised on frozen tissue sections. RTU Vectastain Universal Kit and NovaRED ${ }^{\mathrm{TM}}$ Substrate kit for peroxidase (Vector, Abcys, Paris, France) were used to reveal the primary antibodies anti-MMP-2 (1/100), anti-MMP3 (1/50) and anti-TIMP-1 (1/50), i.e. the same antibodies as those used for immunofluorescence on cell

\begin{tabular}{lcc} 
TABLE 4 & $\begin{array}{l}\text { Number of cycles and PCR conditions for } \\
\text { nonquantitative RT-PCR }\end{array}$ & PCR conditions \\
\hline Cycles n & $\mathrm{B}^{\#}$ \\
MMP-3 & 42 & $\mathrm{~B}^{\text {T }}$ \\
MMP-7 & 40 & $\mathrm{~A}^{\text {* }}$ \\
MMP-9 & 30 & $\mathrm{~B}$ \\
MMP-13 & 42 & $\mathrm{~A}$ \\
MT2-MMP & 30 & \\
\hline
\end{tabular}

MMP: matrix metalloproteinase; MT: membrane type. ${ }^{\#:}: 94^{\circ} \mathrm{C}$ for $15 \mathrm{~s}, 60^{\circ} \mathrm{C}$ for $30 \mathrm{~s}, 72^{\circ} \mathrm{C}$ for $30 \mathrm{~s} ;{ }^{\circ}: 94^{\circ} \mathrm{C}$ for $15 \mathrm{~s}, 68^{\circ} \mathrm{C}$ for $20 \mathrm{~s}, 72^{\circ} \mathrm{C}$ for $10 \mathrm{~s}$

cultures. The slides were counterstained with Mayer's haematoxylin.

In situ zymography was performed as previously described [6, 8]. Serial arterial sections $10 \mu \mathrm{m}$ in thickness were stained with orcein-picro-indigo-carmine to localise collagen and elastin in PAs [6].

\section{Statistical analysis}

Results are expressed as mean \pm SEM. Statistical analysis was performed using the Mann-Whitney U-test, p-values $<0.05$ were considered statistically significant.

\section{RESULTS \\ MMP and TIMP expression in conditioned media of PA-SMCs from controls and patients with IPAH}

In controls, the predominant MMPs were MMP-1, MMP-2 and MMP-3 (fig. 1), whereas MMP-7 and MMP-9 were barely detectable. The level of latent MMP-2 (149.0 $\pm 31.0 \mathrm{ng} \cdot 10^{-5}$ cells) in PA-SMCs was approximately five-times the level of MMP-1 $\left(25.8 \pm 7.4 \mathrm{ng} \cdot 10^{-5}\right.$ cells $)$ and 10-times the level of MMP-3 (15.8 $\pm 4.8 \mathrm{ng} \cdot 10^{-5}$ cells). TIMP-1 was also produced $\left(740 \pm 400 \mathrm{ng} \cdot 10^{-5}\right.$ cells). MMP-7 and TIMP-2 were undetectable using ELISA (data not shown). Consequently, TIMP-2 was assessed by reverse zymography (fig. 2), which detected both TIMP-1 and TIMP-2. In keeping with the ELISA results, TIMP-1 was largely predominant.

Differences in MMP and TIMP expression were found between control and IPAH cells. The IPAH cells showed a significant decrease in MMP-3 production (15.8 \pm 4.8 versus $2.4 \pm 0.7 \mathrm{ng} \cdot 10^{-5}$ cells) and a significant increase in TIMP-1 production $\left(740 \pm 400\right.$ versus $2,700 \pm 600 \mathrm{ng} \cdot 10^{-5}$ cells $)$ as

TABLE 3 Sequence of forward and reverse primers used for nonquantitative RT-PCR amplification

\begin{tabular}{lcc} 
& Forward primers $\left.\mathbf{( 5}^{\prime} \mathbf{- 3} \mathbf{\prime}\right)$ & Reverse primers $\mathbf{( 5}^{\prime} \mathbf{- 3} \mathbf{)}$ \\
\hline MMP-3 & GATCCTTCATTTGGCCATCTCTTC & CTTCCAGTATTTGTCCTCTACAAAGAA \\
MMP-7 & CCCCCTGCATTCAGGAA & TCCTGGCCATCAAATGG \\
MMP-9 & GCGGAGATTGGGACCAGCTGTA & GACGCGCCTGTGTACACCCACA \\
MMP-13 & ATGATCTTAAAGACAGATTCTTCTGG \\
MT2-MMP & CTTGCAGAGATGCAGCGCTTCTAC & TGGGATAACCTTCCAGAATGTCATAA \\
\hline
\end{tabular}

MMP: matrix metalloproteinase; MT: membrane type. 

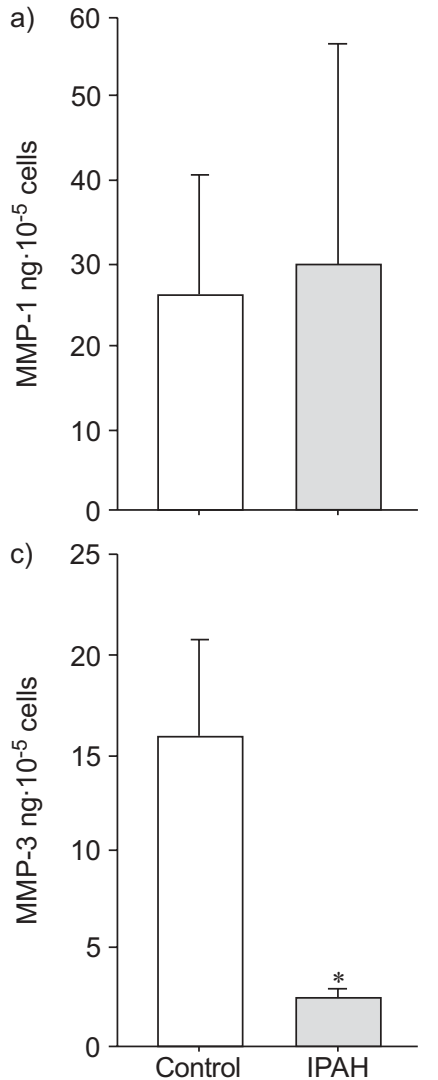
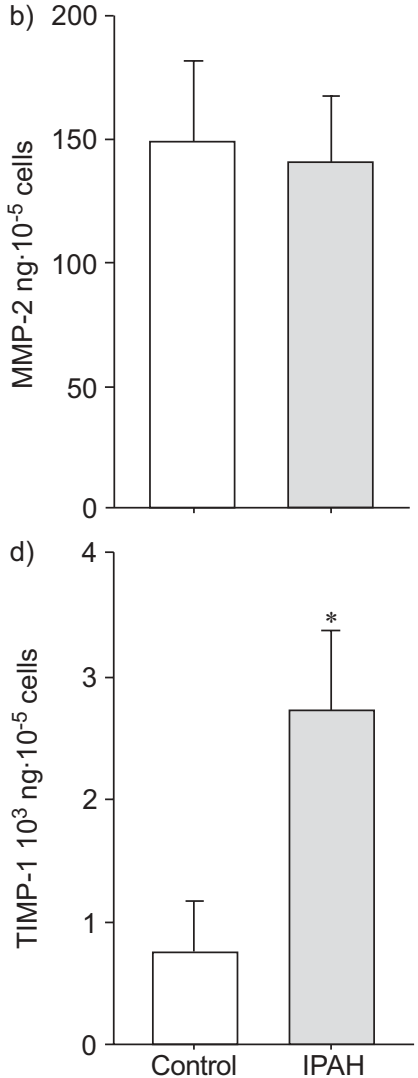

FIGURE 1. ELISA quantification of a) matrix metalloproteinase (MMP)-1, b) MMP-2, c) MMP-3 and d) tissue inhibitor of metalloproteinase (TIMP)-1 in conditioned media of pulmonary artery smooth muscle cells from patients with idiopathic pulmonary arterial hypertension (IPAH) and from controls. Measurements were performed in triplicate for each control $(n=4)$ and IPAH $(n=4)$ specimen. Data are presented as mean \pm SEM. MMP-7 and TIMP-2 were undetectable. *: $p<0.05$.

compared with the control cells. In contrast, no difference was detected between control and IPAH cells for latent MMP-2 $\left(149.0 \pm 31.0\right.$ versus $140.0 \pm 27.1 \mathrm{ng} \cdot 10^{-5}$ cells), for MMP-1 $\left(25.8 \pm 7.4\right.$ versus $29.8 \pm 15.6 \mathrm{ng} \cdot 10^{-5}$ cells; fig. 1$)$ or for TIMP-2 $\left(160 \pm 20\right.$ versus $180 \pm 20 \mathrm{ng} \cdot 10^{-5}$ cells; fig. $\left.2 \mathrm{~b}\right)$.

The anti-MMP-2 antibody used for ELISA recognised only latent MMP-2; therefore, conditioned media were subjected to gelatin zymography to assess MMP-2 activation. Gelatin zymography analysis and densitometry data (fig. 3a and b) showed that active MMP-2 was clearly visible in IPAH cells, but was barely detectable in control cells. As a result, total gelatinase activity was higher in the IPAH cells than in controls. This method also confirmed the ELISA results showing similar latent MMP-2 levels in IPAH and control cells.

MMP-9 was not expressed spontaneously and was induced in low levels by PMA (data not shown) in both IPAH and control cells. Although BMPR-II mutations play a key role in familial $\mathrm{PAH}$ [14], the levels of MMP-9 and of latent and active MMP-2 were not modified by addition of TGF- $\beta$, BMP-4 or BMP-6, three ligands of the TGF- $\beta$-receptor superfamily, or PDGF (data not shown).
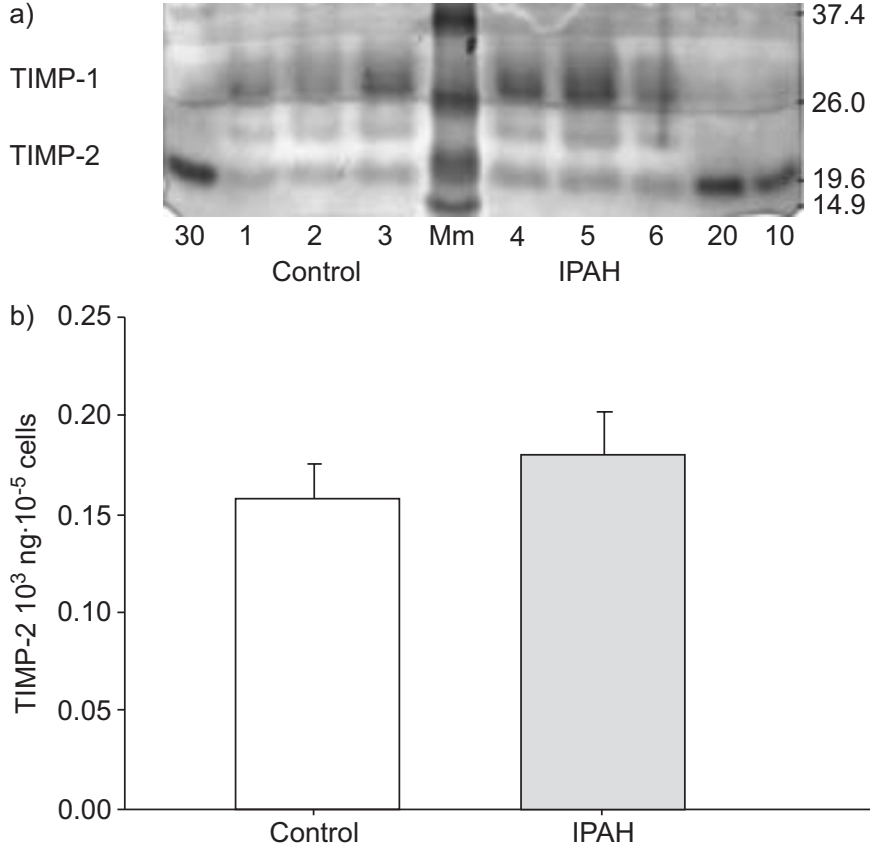

FIGURE 2. Reverse zymography evaluation of tissue inhibitor of metalloproteinase (TIMP)-2 in conditioned media of pulmonary artery smooth muscle cells from patients with idiopathic pulmonary arterial hypertension (IPAH) and from controls. a) Lanes 1-3 show three different controls and lanes 4-6 show IPAH cell cultures. Molecular mass standards are indicated on the right. The molecular masses of TIMP-1 $(29 \mathrm{kDa})$ and TIMP-2 $(21 \mathrm{kDa})$ are shown on the left. Three amounts of recombinant human TIMP-2 (30,20 and $10 \mathrm{ng})$ were used to establish a standard curve. b) Data are presented as mean \pm SEM.

Presence of MT1-MMP was evaluated by Western blot. This showed three MT1-MMP forms of 60, 50 and $37 \mathrm{kDa}$, as previously described [15], in both IPAH and control cells (data not shown).

\section{MMP and TIMP transcript expression by PA-SMCs from controls and IPAH patients}

Quantitative RT-PCR showed no difference between IPAH and control cells for MMP-1 transcript expression. Trends were found towards increases in MMP-2, MT1-MMP, TIMP-1 and TIMP-2 transcript expression in IPAH (fig. 4). MT2-MMP transcript levels were similar in control and IPAH cells (data not shown).

No MMP-9 or MMP-7 mRNAs were found, even with up to 40 PCR cycles. Despite the use of 42 PCR cycles, MMP-3 mRNA was detected in only four out of six controls and two out of five IPAH patients (fig. 5), and quantitative RT-PCR could not be performed. Furthermore, when detected, MMP-3 mRNA bands were less marked in IPAH cells than in control cells.

\section{MMP and TIMP immunostaining of cultured PA-SMCs}

Representative photographs of immunofluorescence studies are shown in figure 6. Quantification (table 5) found overexpression of MMP-2 and TIMP-1 and reduced expression of MMP-3 in PA-SMCs from IPAH specimens, with similar levels of both MT1-MMP and TIMP-2. 
a)

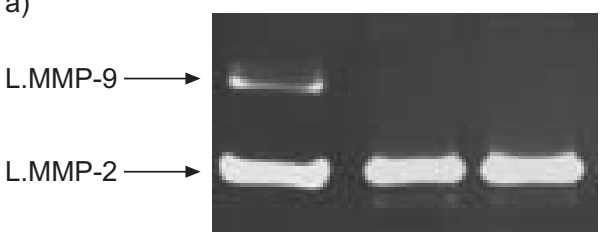

b)

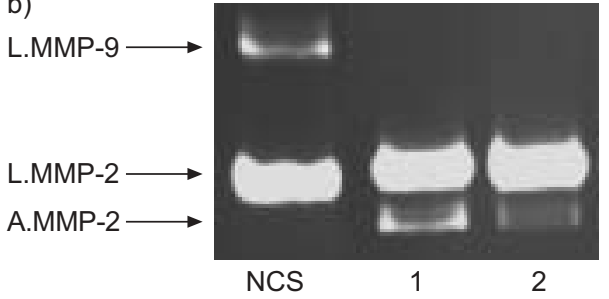

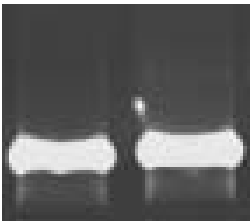

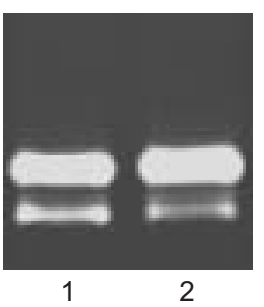

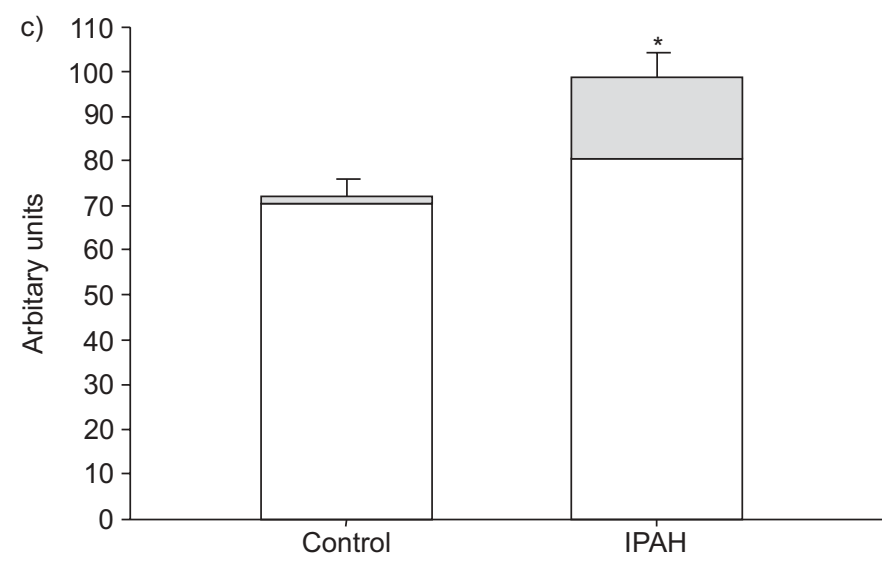

FIGURE 3. Expression ( $a$ and b) and quantification (c) of gelatinase produced by pulmonary artery smooth muscle cells from patients with idiopathic pulmonary arterial hypertension (IPAH) and from controls. a) Two different control-cell cultures and b) IPAH-cell cultures from two different patients are shown. Lane 1 shows the unstimulated condition serum-free medium and lane 2 shows stimulation by transforming growth factor- $\beta\left(10 \mathrm{ng} \cdot \mathrm{mL}^{-1}\right)$. As a control for the size and relative quantification of gelatinolysis bands, $5 \%$ new calf serum medium (NCS) was used. L.: latent; MMP: matrix metalloproteinase; A.: active. c) Data are presented as mean \pm SEM amounts of L.MMP-2 ( $\square$ ) and A.MMP-2 ( $\square$ ) produced by unstimulated cells from controls $(n=5)$ and patients with IPAH $(n=5)$. The values for each MMP band were normalised for the value obtained with NCS. *: $p<0.05$.

\section{Immunostaining and gelatinolytic activity in PA walls}

In situ, gelatinolytic activity and MMP-2 immunostaining were colocalised and predominated in the media (figs 7 and 8). MMP-2 immunolocalisation was found in SMCs and was also associated with elastic fibres in both IPAH and control specimens. In IPAH arteries, high gelatinolytic activity and MMP-2 immunostaining were found all along the inner elastic lamina up to the break in this structure. Interestingly, at these sites, a marked change in SMC orientation was noted (arrow head in fig. 8f), consistent with SMC migration from the media to the neo-intima. MMP-3 staining in the media was weak or absent in control specimens, and absent in IPAH specimens. TIMP-1 immunostaining was diffuse and strong in both control and IPAH arteries.

a)

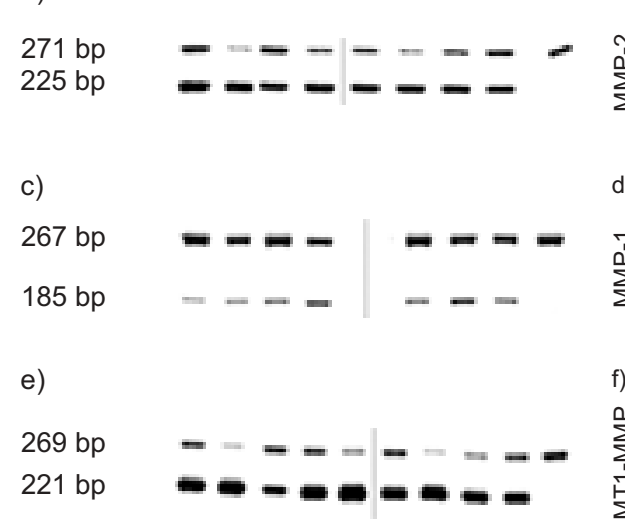

g)

$271 \mathrm{bp}$

$170 \mathrm{bp}$

i)

$269 \mathrm{bp}$

$155 \mathrm{bp}$
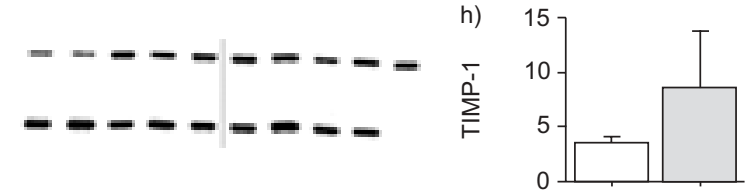

j)
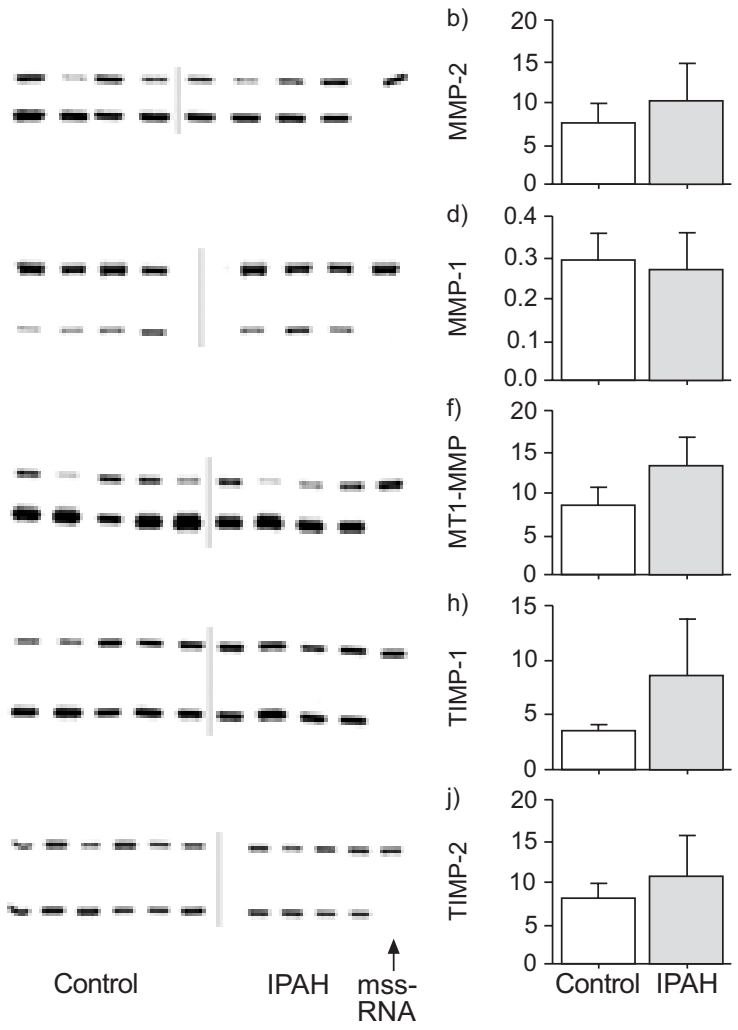

FIGURE 4. Electrophoretic (a, c, e, g, i) and scanning densitometry (b, d, f, h, j) of quantitative RT-PCR products for matrix metalloproteinase (MMP)-2 $(a, b)$, MMP-1 (c, d), membrane type (MT)1-MMP (e, f), tissue inhibitor of metalloproteinase (TIMP)-1 $(\mathrm{g}, \mathrm{h}$ ) and TIMP-2 (i, j) in pulmonary artery smooth muscle cells from controls and idiopathic pulmonary arterial hypertension (IPAH) patients. a, c, e, g, i) The bands at the top show amplified multiple synthetic internal stranded RNA (mssRNA) and the bands at the bottom show amplified mRNAs. b, d, f, h, j) Steadystate levels of mRNAs expressed as the ratio over 28s mRNA expression ((MMPX/ mssRNAx)/(28s/mssRNA28s)). Data are presented as mean \pm SEM.

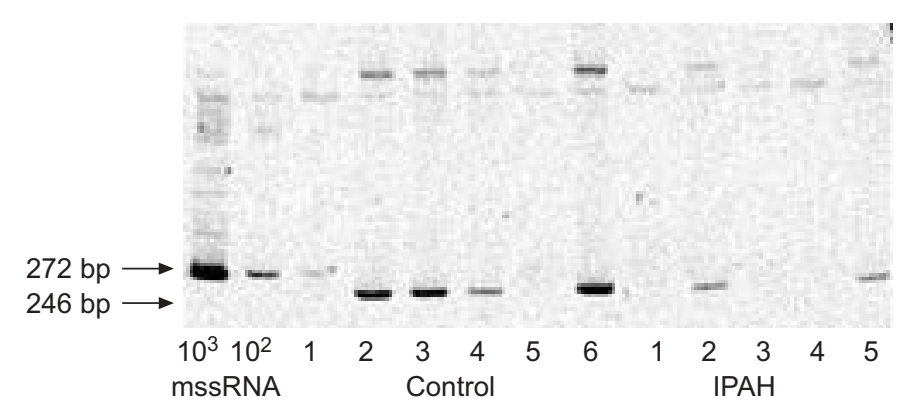

FIGURE 5. Electrophoresis of the RT-PCR products for matrix metalloproteinase (MMP)-3 from pulmonary artery smooth muscle cells from controls $(n=8)$ and idiopathic pulmonary arterial hypertension (IPAH) patients $(n=7)$. The size of RT-PCR products of multiple synthetic internal stranded RNA (mssRNA; 272 bp) and MMP-3 mRNA (246 bp) are indicated.

\section{DISCUSSION}

Marked differences between IPAH patients and controls were found in the current study. In vitro, TIMP-1 was overexpressed and MMP-3 under-expressed by IPAH cells, 

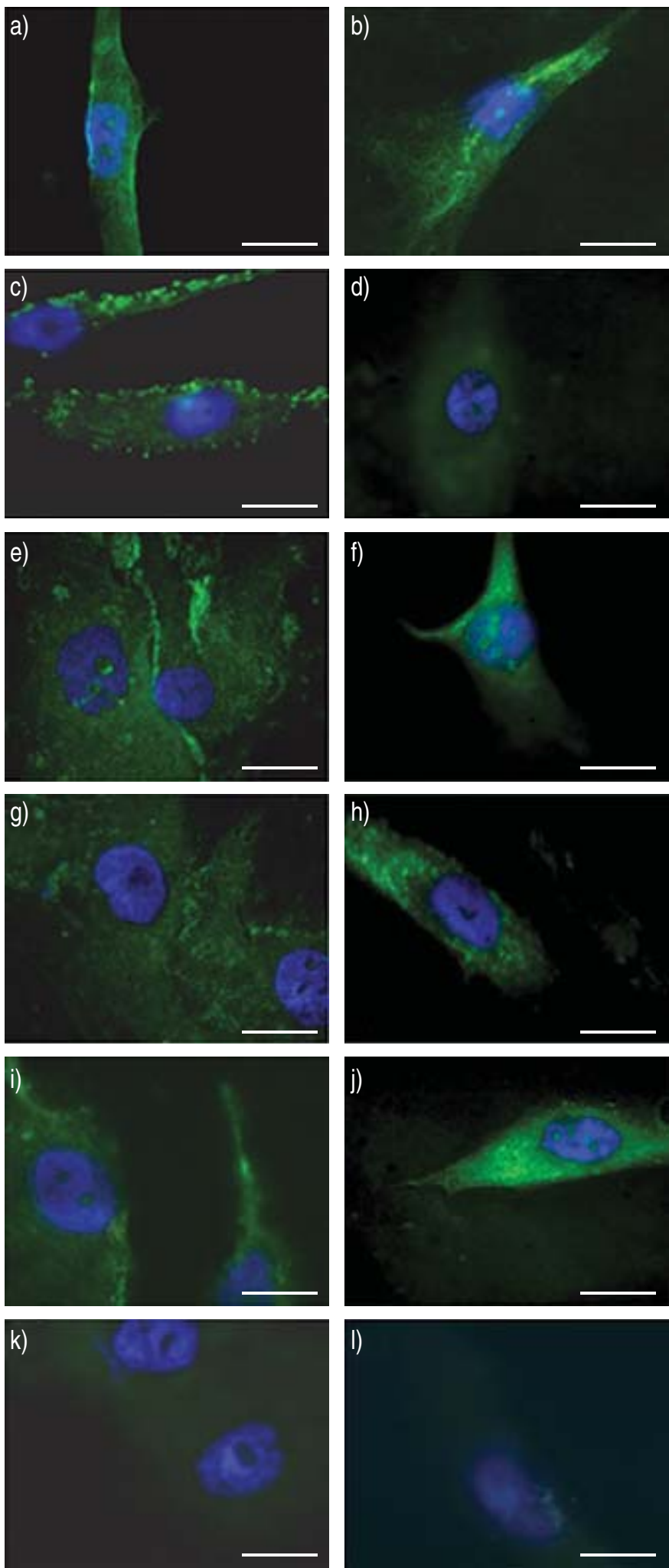

FIGURE 6. Matrix metalloproteinase (MMP)-2 (a, b), MMP-3 (c, d), membrane type 1-MMP (e, f), tissue inhibitor of metalloproteinase (TIMP)-2 ( $g, h)$ and TIMP-1 $(i, j)$ expression by pulmonary artery smooth muscle cells, as evaluated by immunofluorescence. Cells are shown from controls $(a, c, e, g, i, k)$ and patients with idiopathic pulmonary artery hypertension (b, d, f, h, j, l). k, l) correspond to negative controls. Scale bars $=5 \mu \mathrm{m}$.
TABLE 5 Immunofluorescence quantification of MMP-2, MMP-3, MT1-MMP, TIMP-2 and TIMP-1 in cultured pulmonary artery smooth muscle cells from patients with IPAH and from controls

\begin{tabular}{lrc} 
& Control & IPAH \\
\hline MMP-2 & $13.0 \pm 0.3$ & $17.5 \pm 0.4^{*}$ \\
MMP-3 & $10.8 \pm 0.2$ & $2.7 \pm 0.2^{*}$ \\
MT1-MMP & $7.9 \pm 0.3$ & $10.3 \pm 1.0$ \\
TIMP-1 & $16.4 \pm 0.2$ & $24.5 \pm 0.2^{*}$ \\
TIMP-2 & $7.8 \pm 0.9$ & $10.7 \pm 0.4$ \\
\hline
\end{tabular}

Data are presented as mean \pm SEM. IPAH: idiopathic pulmonary arterial hypertension; MMP: matrix metalloproteinase; MT: membrane type; TIMP: tissue inhibitors of metalloproteinase. * $p<0.05$

whereas MMP-1 expression was similar in the two groups. Total MMP-2 overexpression was also found, with a greater amount of the active form in IPAH cells as compared with controls. In situ studies showed gelatinolytic activity in tissue sections and strong MMP-2 immunostaining along the inner elastic lamina up to the lamina break. TIMP-1 immunostaining was found in both control and IPAH arteries, whereas MMP-3 staining was detected only in the media of a few control specimens. This evidence, indicating severe disruption of the TIMP-MMP balance, is consistent with a major role for this system in pulmonary vascular remodelling in IPAH.

A major finding from the present study is the marked increase in TIMP-1 protein in PA-SMCs from IPAH patients. Excessive TIMP levels have been reported in fibrotic diseases characterised by ECM accumulation, such as hepatic [16] and pulmonary fibrosis [17]. TIMP-1 is more widely distributed than the other TIMPs and inhibits the activity of all active MMPs [5]. The current findings are in keeping with histological studies in human PAs showing intimal fibrosis and medial hypertrophy corresponding to accumulation of ECM, particularly collagen $[1,18]$.

This study also found lower MMP-3 levels in IPAH-conditioned media, with a decrease in MMP-3 mRNAs, suggesting transcriptional regulation. The low MMP-3 expression in this study is consistent with an earlier report of weak MMP-3 staining in myofibroblasts from remodelled PAs and negative MMP-3 staining in mature plexiform lesions [19]. MMP-3 is a key member of the MMP family and is characterised by broad substrate specificity, including several collagens, proteoglycan, laminin and fibronectin [5]. In addition, MMP-3 can activate latent forms of other MMPs, such as MMP-1 and MMP-8, a property that gives MMP-3 a crucial role in connective tissue remodelling [5]. The TIMP-1-MMP-3 imbalance found in IPAH cells in the present study would be expected to promote ECM accumulation.

Zymograms showed significantly higher MMP-2 levels in the IPAH cells related to the presence of the active enzyme form, which was barely detectable in control cells. This result is consistent with previous studies showing increased levels and activation of MMP-2 in PAs from experimental PAH models [8]. An important issue is activation of latent MMP-2, which 

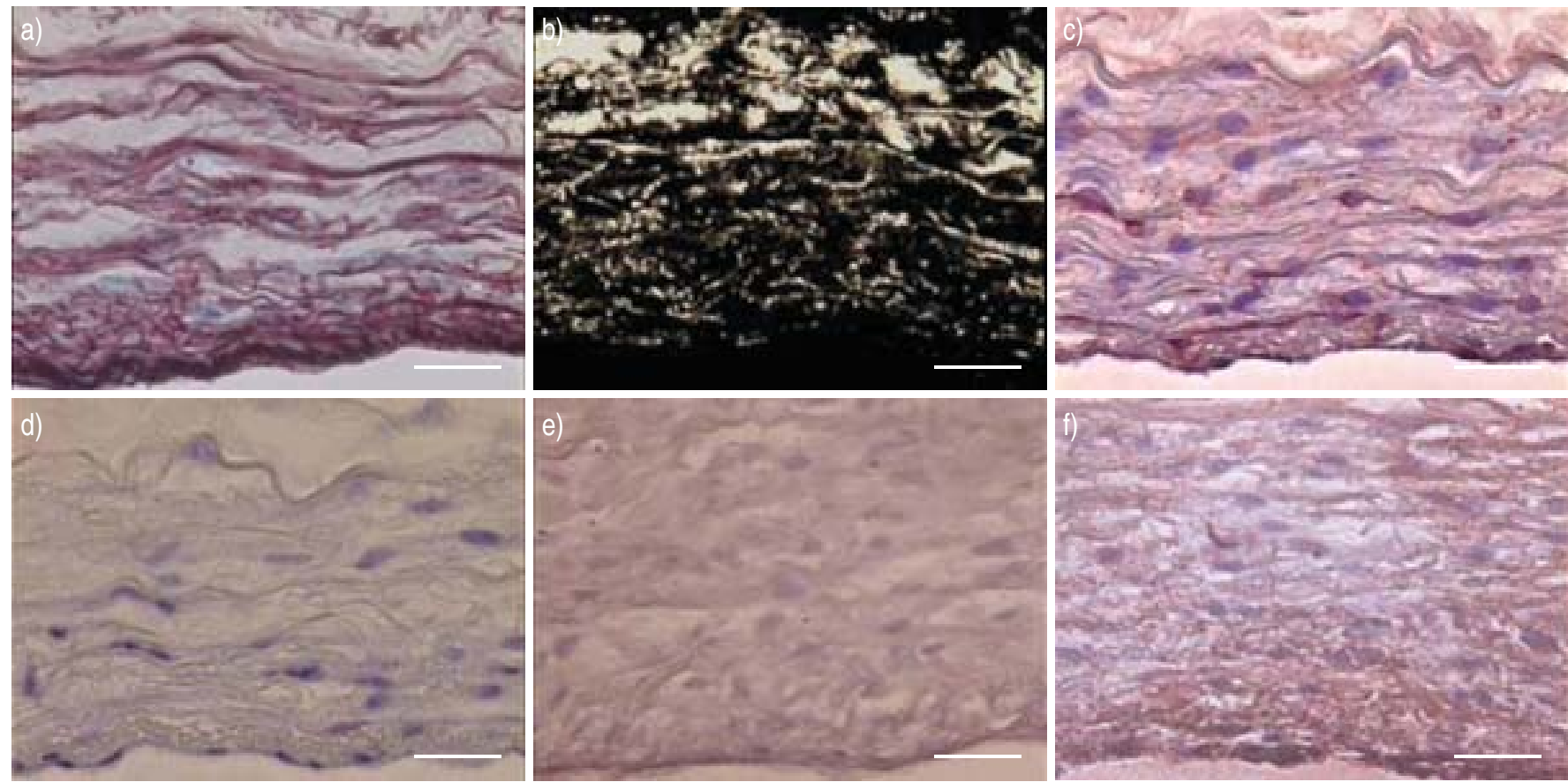

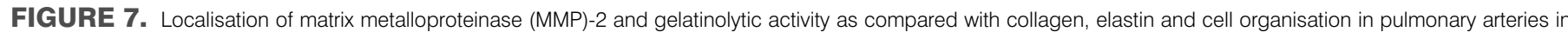

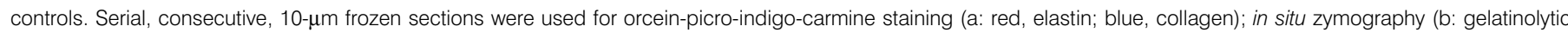

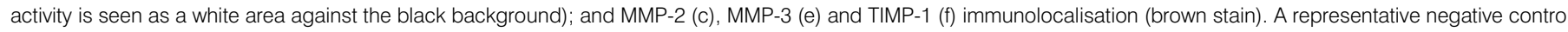
without antibody is shown in (d). Scale bars $=30 \mu \mathrm{m}$.

can occur via several pathways. Latent MMP-2 activation by MMP-7 [20] probably did not occur in this study, as no MMP-7 expression was detected. MMP-2 can also be activated via the MMP-2/MT-1MMP/TIMP-2 complex [5]. However, production of MT1-MMP (Western blot, data not shown) and TIMP-2 (fig. 2) was similar in IPAH and control cells. The two other known mechanisms of MMP-2 activation are MMP-2 autocatalytic activation by another molecule of active MMP-2, which must be tethered to the cell surface [5], and TIMP-2independent activation [9] involving MT2-MMP. The second mechanism may activate MMP-2 in processes and tissues characterised by induction of MT2-MMP and low levels of TIMP-2 [9], a situation suggested by the results of the present study.

Demonstration of a TIMP-1-MMP imbalance conducive to ECM accumulation does not rule out a role for active MMP-2 in IPAH. Proteolysis may be effective in limited areas even when TIMP levels are high in the extracellular milieu, because MMP-2 tethering and activation at the cell surface focuses the catalytic activity on limited targets on the cell membrane. This hypothesis is supported by the immunohistology and in situ zymography data, which clearly show that gelatinolytic activity colocalised with MMP-2 immunostaining in arteries from IPAH patients. This pattern of MMP and TIMP expression, characterised by increased TIMP-1 levels coexisting with evidence of ECM degradation, has been found in other fibrotic diseases, such as adult respiratory distress syndrome (ARDS). In bronchoalveolar lavage (BAL) fluid from ARDS patients, TIMP-1 levels were significantly higher than in healthy controls [21]. Despite the high TIMP-1 levels, ECM degradation by MMPs is suggested by the presence of active
MMP-2 in epithelial lining fluid [22] and of basement membrane disruption markers in BAL fluid [23] of ARDS patients. Altogether, these data suggest that a TIMP-1-MMP imbalance promoting ECM accumulation within the interstitial tissue may coexist with the presence of active MMP-2 confined to the cell surface.

In IPAH, disruption of the internal elastic lamina, ECM disorganisation and SMC migration are strong arguments supporting a direct role for active MMP-2. This enzyme not only degrades nonfibrillar collagen, but also cleaves elastin [5] Elastin fragmentation has been shown to be an early pulmonary vascular abnormality in patients with congenital heart defects and PAH [2]. Elastinolytic activities have also been ascribed to endogenous vascular elastase [24, 25]. In addition, elastinolytic properties of MMP-2 should also be taken into account [26]. Moreover, latent-MMP-2 may both bind to elastin and undergo auto-activation, subsequently degrading elastin [27]. The results presented here are consistent with previous data, as in situ zymography and MMP-2 immunolocalisation showed colocalisation of gelatinolytic activities and MMP-2 along elastic fibres. Also, active MMP-2 may contribute not only to ECM remodelling but also to important processes in IPAH, such as SMC migration and proliferation [10, 28].

A number of methodological issues deserve discussion. First, the number of specimens was small, as the authors studied human tissue and the prevalence of IPAH in humans is low $(0.5$ per $1,000,000)$. Secondly, the controls were specimens from patients who had surgery for lung cancer. Many studies have found increases in MMP and TIMP in cancer tissue as 

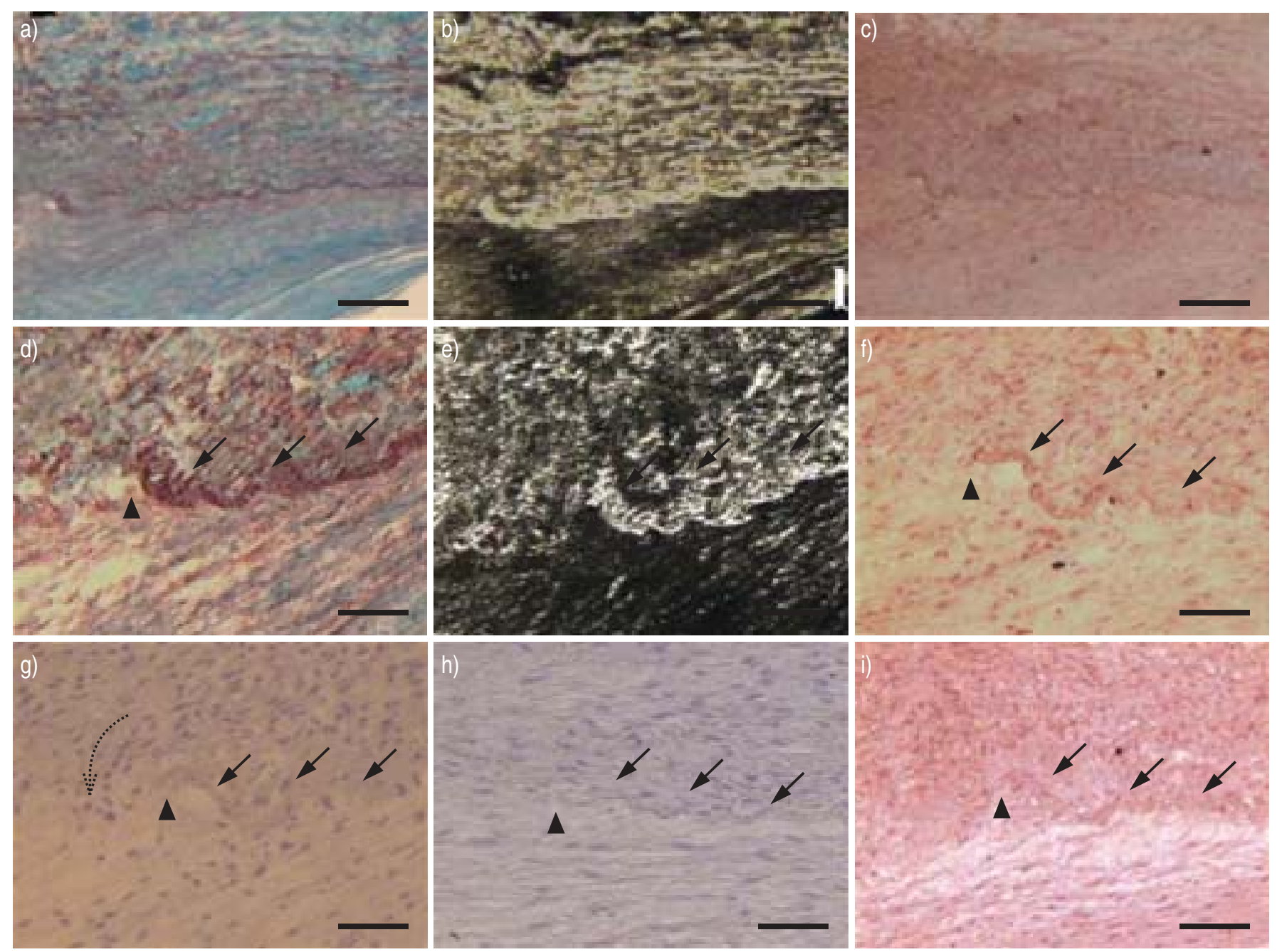

FIGURE 8. Localisation of matrix metalloproteinase (MMP)-2 and gelatinolytic activity as compared with collagen, elastin and cell organisation in pulmonary arteries (PAs) from idiopathic pulmonary hypertension. a-c) PAs $\times 100$ magnification. $d-i)$ The same arteries at $\times 400$ magnification. Serial, consecutive, 10- $\mu$ m frozen sections were used for orcein-picro-indigo-carmine staining ( $\mathrm{a}, \mathrm{d}$ : red, elastin; blue, collagen); in situ zymography (b, e: gelatinolytic activity is seen as a white area against the black background); MMP-2 (c, f), MMP-3 (h) and tissue inhibitor of metalloproteinase-1 (j) immunolocalisation (brown stain); g) shows a representative negative control without primary antibody, revealed with RTU Vectastain Universal Kit, NovaRED ${ }^{\mathrm{TM}}$ substrate kit for peroxidase and counterstained with Mayer's haematoxylin. $\left.d-j\right)$ Arrows point to the inner elastic lamina and the arrowheads to the break in the lamina. The dotted arrow in g) indicates a shift in cell orientation at the inner elastic lamina break. Scale bars $=260 \mu \mathrm{m}(\mathrm{a}-\mathrm{c})$ and $85 \mu \mathrm{m}(\mathrm{d}-\mathrm{i})$.

compared with adjacent normal tissue [29]. In the present study, the PAs were dissected out several centimetres away from the tumours. Moreover, most of the data from this study came from cultured SMCs, which were not primarily involved in the tumoural angiogenic process. Finally, the histological and immunostaining data were obtained from fairly large PAs. However, most of the data were obtained from SMCs in vitro, and there were clear differences between patient SMCs and control SMCs that persisted over time. A previous study in rats found that MMP expression was similar in proximal and distal arteries [8].

In conclusion, this in situ and in vitro study of human pulmonary arteries found an imbalance between matrix metalloproteinase and tissue inhibitors of metalloproteinase-1 and an increase in active matrix metalloproteinase- 2 in idiopathic pulmonary arterial hypertension cells as compared with controls. The imbalance in favour of tissue inhibitor of metalloproteinase-1 may lead to extracellular matrix accumulation. Active matrix metalloproteinase- 2 confined to the cell may contribute to other processes, such as smooth muscle cell migration and proliferation. Whether the abnormalities found in the current study are potential therapeutic targets deserves investigation.

\section{REFERENCES}

1 Archer S, Rich S. Primary pulmonary hypertension: a vascular biology and translational research "Work in progress". Circulation 2000; 102: 2781-2791.

2 Rabinovitch M, Bothwell T, Hayakawa BN, et al. Pulmonary artery endothelial abnormalities in patients with congenital heart defects and pulmonary hypertension. A correlation of light with scanning electron 
microscopy and transmission electron microscopy. Lab Invest 1986; 55: 632-653.

3 Cowan KN, Jones PL, Rabinovitch M. Elastase and matrix metalloproteinase inhibitors induce regression, and tenascin- $C$ antisense prevents progression, of vascular disease. J Clin Invest 2000; 105: 21-34.

4 Cowan KN, Heilbut A, Humpl T, Lam C, Ito S, Rabinovitch M. Complete reversal of fatal pulmonary hypertension in rats by a serine elastase inhibitor. Nat Med 2000; 6: 698-702.

5 Overall CM, Lopez-Otin C. Strategies for MMP inhibition in cancer: innovations for the post-trial era. Nat Rev Cancer 2002; 2: 657-672.

6 Vieillard-Baron A, Frisdal E, Eddahibi S, et al. Inhibition of matrix metalloproteinases by lung TIMP-1 gene transfer or doxycycline aggravates pulmonary hypertension in rats. Circ Res 2000; 87: 418-425.

7 Vieillard-Baron A, Frisdal E, Raffestin B, et al. Inhibition of matrix metalloproteinases by lung TIMP-1 gene transfer limits monocrotaline-induced pulmonary vascular remodeling in rats. Hum Gene Ther 2003; 14: 861-869.

8 Frisdal E, Gest V, Vieillard-Baron A, et al. Gelatinase expression in pulmonary arteries during experimental pulmonary hypertension. Eur Respir J 2001; 18: 838-845.

9 Morrison CJ, Butler GS, Bigg HF, Roberts CR, Soloway PD, Overall CM. Cellular activation of MMP-2 (gelatinase A) by MT2-MMP occurs via a TIMP-2-independent pathway. J Biol Chem 2001; 276: 47402-47410.

10 Uzui H, Lee JD, Shimizu H, Tsutani H, Ueda T. The role of protein-tyrosine phosphorylation and gelatinase production in the migration and proliferation of smooth muscle cells. Atherosclerosis 2000; 149: 51-59.

11 Eddahibi S, Humbert M, Fadel E, et al. Serotonin transporter overexpression is responsible for pulmonary artery smooth muscle hyperplasia in primary pulmonary hypertension. J Clin Invest 2001; 108: 1141-1150.

12 Sheares KK, Jeffery TK, Long L, Yang X, Morrell NW. Differential effects of TGF- $\beta_{1}$ and BMP-4 on the hypoxic induction of cyclooxygenase-2 in human pulmonary artery smooth muscle cells. Am J Physiol Lung Cell Mol Physiol 2004; 287: L919-L927.

13 Lambert CA, Colige AC, Munaut C, Lapiere CM, Nusgens BV. Distinct pathways in the over-expression of matrix metalloproteinases in human fibroblasts by relaxation of mechanical tension. Matrix Biol 2001; 20: 397-408.

14 Lane KB, Machado RD, Pauciulo MW, et al. Heterozygous germline mutations in BMPR2, encoding a TGF- $\beta$ receptor, cause familial primary pulmonary hypertension. The International PPH Consortium. Nat Genet 2000; 26: 81-84.

15 Stanton H, Gavrilovic J, Atkinson SJ, et al. The activation of ProMMP-2 (gelatinase A) by HT1080 fibrosarcoma cells is promoted by culture on a fibronectin substrate and is concomitant with an increase in processing of MT1-MMP (MMP-14) to a 45 kDa form. J Cell Sci 1998; 111: 2789-2798.
16 Arthur MJ. Fibrogenesis II. Metalloproteinases and their inhibitors in liver fibrosis. Am J Physiol Gastrointest Liver Physiol 2000; 279: Suppl. 2, G245-G249.

17 Corbel M, Belleguic C, Boichot E, Lagente V. Involvement of gelatinases (MMP-2 and MMP-9) in the development of airway inflammation and pulmonary fibrosis. Cell Biol Toxicol 2002; 18: 51-61.

18 Voelkel NF, Tuder RM. Cellular and molecular mechanisms in the pathogenesis of severe pulmonary hypertension. Eur Respir J 1995; 8: 2129-2138.

19 Matsui K, Takano Y, Yu ZX, et al. Immunohistochemical study of endothelin-1 and matrix metalloproteinases in plexogenic pulmonary arteriopathy. Pathol Res Pract 2002; 198: 403-412.

20 Crabbe T, Smith B, O'Connell J, Docherty A. Human progelatinase A can be activated by matrilysin. FEBS Lett 1994; 345: 14-16.

21 Ricou B, Nicod L, Lacraz S, Welgus HG, Suter PM, Dayer JM. Matrix metalloproteinases and TIMP in acute respiratory distress syndrome. Am J Respir Crit Care Med 1996; 154: 346-352.

22 Delclaux C, d'Ortho MP, Delacourt C, et al. Gelatinases in epithelial lining fluid of patients with adult respiratory distress syndrome. Am J Physiol 1997; 272: L442-L451.

23 Kondoh Y, Taniguchi H, Taki F, Takagi K, Satake T. 7S collagen in bronchoalveolar lavage fluid of patients with adult respiratory distress syndrome. Chest 1992; 101: 1091-1094.

24 Jacob MP, Bellon G, Robert L, et al. Elastase-type activity associated with high density lipoproteins in human serum. Biochem Biophys Res Commun 1981; 103: 311-318.

25 Zhu L, Wigle D, Hinek A, et al. The endogenous vascular elastase that governs development and progression of monocrotaline-induced pulmonary hypertension in rats is a novel enzyme related to the serine proteinase adipsin. $J$ Clin Invest 1994; 94: 1163-1171.

26 Senior RM, Griffin GL, Fliszar CJ, Shapiro SD, Goldberg GI, Welgus HG. Human 92- and 72-kilodalton type IV collagenases are elastases. J Biol Chem 1991; 266: 7870-7875.

27 Emonard H, Hornebeck W. Binding of $92 \mathrm{kDa}$ and $72 \mathrm{kDa}$ progelatinases to insoluble elastin modulates their proteolytic activation. Biol Chem 1997; 378: 265-271.

28 George SJ, Johnson JL, Angelini GD, Newby AC, Baker AH. Adenovirus-mediated gene transfer of the human TIMP-1 gene inhibits smooth muscle cell migration and neointimal formation in human saphenous vein. Hum Gene Ther 1998; 9: 867-877.

29 Schutz A, Schneidenbach D, Aust G, Tannapfel A, Steinert M, Wittekind C. Differential expression and activity status of MMP-1, MMP-2 and MMP-9 in tumor and stromal cells of squamous cell carcinomas of the lung. Tumour Biol 2002; 23: 179-184. 